\title{
ARTICLE
}

Molecular Diagnostics

\section{The combination of complex karyotype subtypes and IGHV mutational status identifies new prognostic and predictive groups in chronic lymphocytic leukaemia}

\author{
Andrea Visentin ${ }^{1,2}$, Laura Bonaldi ${ }^{3}$, Gian Matteo Rigolin ${ }^{4}$, Francesca Romana Mauro ${ }^{5}$, Annalisa Martines ${ }^{3}$, Federica Frezzato ${ }^{1,2}$, \\ Silvia Imbergamo ${ }^{1,2}$, Edoardo Scomazzon ${ }^{1,2}$, Stefano Pravato ${ }^{1,2}$, Maria Antonella Bardi ${ }^{4}$, Maurizio Cavallari ${ }^{4}$, Eleonora Volta ${ }^{4}$, \\ Francesco Cavazzini ${ }^{4}$, Maurizio Nanni ${ }^{5}$, Ilaria Del Giudice ${ }^{5}$, Monica Facco ${ }^{1,2}$, Anna Guarini ${ }^{5}$, Gianpietro Semenzato ${ }^{1,2}$, Robin Foà ${ }^{5}$, \\ Antonio Cuneo ${ }^{4}$ and Livio Trentin ${ }^{1,2}$
}

BACKGROUND: Complex karyotype (CK) is a heterogeneous category with a negative impact in chronic lymphocytic leukaemia (CLL). Our group has recently reported that CK patients with major structural abnormalities (i.e. CK2) are characterised by a worse prognosis, as compared to other lesions within CK(CK1).

METHODS: We performed a multicentre retrospective study to test whether the combination of CK subtypes with IGHV status could be a relevant prognostic and predictive tool.

RESULTS: Among 522 patients 13\% harboured CK2, 41\% CK1 and/or U-IGHV (U-CK1) and 46\% M-IGHV without any CK subtypes (M-noCK). After a median follow-up of 5.8 years, CK2 patients had the shortest TTFT ( 5 -year TTFT $31 \%, 39$ and $81 \%, p<0.0001$ ) and OS (5-year OS $67 \%, 85$ and 93\%, $p<0.0001)$ as compared to U-CK1 or M-noCK cases, regardless of TP53 abnormalities. CK2 patients also had the worst outcome after chemoimmunotherapy. In fact, the median TTNT after FCR or BR was 1.86 and 4.79 years for CK2 and U-CK1, but not reached for M-noCK patients $(p<0.0005)$.

CONCLUSIONS: We herein suggest that the combined assessment of the IGHV mutational status and CK subtypes refines the prognostication of CLL, allowing to identify M-IGHV patients without any CK subtypes who are characterised by an indolent disease and excellent outcome after chemoimmunotherapy.

British Journal of Cancer (2019) 121:150-156; https://doi.org/10.1038/s41416-019-0502-x

\section{BACKGROUND}

Chronic lymphocytic leukaemia (CLL), the most common leukaemia in western countries, is a remarkably heterogeneous disease, with some patients never requiring treatment and others with a highly aggressive and/or rapidly progressive clinical course. ${ }^{1,2}$ The three most important CLL prognostic factors, variables capable of defining subjects at a higher risk of progression or death, and predictive genetic markers, which identify subjects who will relapsed earlier after treatments, are represented by leukemic cell cytogenetic assessed by fluorescence in situ hybridisation (FISH), TP53 abnormalities, including mutations and deletions, and the mutational status of the variable region of the immunoglobulin heavy chain (IGHV) genes. ${ }^{3}$

Recent studies have shown that current FISH analysis, according to Dohner's hierarchical model, underestimates the true genetic complexity revealed by chromosome banding analysis. In fact, $22-36 \%$ of CLL cases with "normal" FISH carry chromosomal aberration at karyotype. In particular, complex karyotype (CK), defined by the presence of at least three chromosome lesions in the same clone, is detectable in $14-34 \%$ of CLL cases $^{4-7}$ and is emerging as a new negative prognostic biomarker associated with an adverse outcome $\mathrm{e}^{4,8}$ and worse response to chemoimmunotherapy ${ }^{5,9}$ as well as to novel agents, ${ }^{10,11}$ regardless of the CLL-IPI score, unmutated IGHV genes and $11 \mathrm{q} / 17 \mathrm{p}$ deletions. ${ }^{6}$ However, the CK itself is a heterogeneous quantitative and qualitative cytogenetic category that includes numerical (i.e. monosomies and trisomies) and structural abnormalities (i.e. balanced and unbalanced translocations, marker chromosomes, isochromosomes, deletions, insertions and additions). Rigolin et al. recently demonstrated that among 90 CK cases the presence of major structural abnormalities at CLL diagnosis identifies a subset of patients with a poor outcome and distinct mRNA expression profile. ${ }^{12}$ However, it is unknown whether the prognostic strength of CK could be improved when combined with a stable marker such as the IGHV mutational status, and whether this approach could help to identify patients who can gain the maximum benefit from chemoimmunotherapy.

In this multicentre retrospective study, we demonstrated that in 522 CLL patients the combination of CK subtypes and IGHV status provides relevant prognostic data, allowing to refine the prognostic stratification of CLL patients, and to identify M-IGHV patients

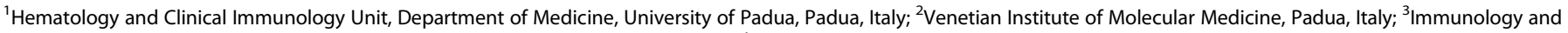

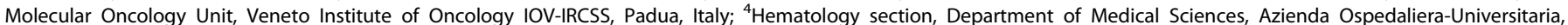
Arcispedale S. Anna, University of Ferrara, Ferrara, Italy and ${ }^{5}$ Hematology division, Department of Translational and Precision Medicine, "Sapienza" University, Rome, Italy Correspondence: Livio Trentin (livio.trentin@unipd.it) 
without any CK subtypes who are characterised by an indolent disease and excellent outcome after chemoimmunotherapy.

\section{METHODS}

Study design

Inclusion criteria for this study were diagnosis of CLL according to the 2008 iwCLL criteria, ${ }^{13}$ age $>18$ years and chromosome banding analysis performed within 1 year from diagnosis. Data included in the comparative analysis were gender, age, Binet stage, $^{13}$ need for chemotherapy, CD38 expression (performed as previously reported ${ }^{2}$ with a cut-off value of $\left.30 \%\right)$, cytogenetics detected by fluorescence in situ hybridisation (FISH), ${ }^{14}$ IGHV mutational analysis ${ }^{15}$ and TP53 abnormalities including gene deletions or mutations. ${ }^{16}$ The primary endpoint was the impact of the combination of CK subtypes with IGHV status on the overall survival (OS) of patients. The correlation with clinico-biological variables and its impact on time to first treatment (TTFT) and relapse after chemoimmunotherapy were considered as secondary endpoints. This study was approved by the local research ethics committee and informed consent was obtained from all patients.

\section{Chromosome banding analysis}

Cytogenetic analysis was performed on peripheral blood after a $72 \mathrm{~h}$ exposure of $500 \mu \mathrm{M}$ CpG ODN DSP30 (Roche, Risch, CH) mitogen $+20 \mathrm{U} / \mathrm{mL}$ IL2 (Roche). Cultures were exposed overnight to $0.1 \mu \mathrm{g} / \mathrm{mL}$ colcemid (Gibco Karyomax Colcemid, ThermoFisher, Waltham, MA USA) to obtain metaphases and then they were harvested following standard procedures. Karyotype was described after the analysis of at least 25 G-banded metaphases using the IKAROS software (MetasYstems, Altlhusseim, Germany), according to International guidelines (ISCN 2016). Complex karyotype (CK) was defined by the presence of three or more chromosome abnormalities in the same clone. ${ }^{4,6,17,18}$ Moreover, based on the type of chromosome changes among CK, we termed Type-2 CK (CK2) those cases with major structural rearrangements (i.e. unbalanced translocations, chromosomes addition, insertion, duplications, ring, dicentric and marker chromosomes). ${ }^{12}$ Whereas, complex karyotypes with balanced translocations, deletions, monosomies or trisomies were called as type 1 (CK1). ${ }^{12}$

IGHV mutational status

Analysis of the IGHV mutational status was performed within 12 months from diagnosis on peripheral blood CLL cells from fresh samples or frozen purified CLL cells harvested in DMSO. RNA was extracted from $2 \times 10^{6}$ B cells using the RNeasy ${ }^{\mathrm{TM}}$ Total RNA kit (Qiagen, Hilgen, Germany) and reverse transcribed using the SuperScript ${ }^{\mathrm{TM}}$ Preamplification System for first-strand CDNA synthesis (Life Technologies, Carlsbad, CA). The CLL B-cell HV gene family was assigned as previously described. ${ }^{19,20}$ HV gene sequences were determined by amplifying $5 \mu \mathrm{l}$ of the original CDNA using the appropriate HV leader and HC primers. PCR products were sequenced directly after purification with Wizard PCR Preps (Promega, Madison, WI) using an automated genetic analyser (3130 ABI Applied Biosystems, Foster City, CA, USA). Sequences were analysed using the IMGT/VQUEST and BLAST softwares $^{21}$ to detect VDJ junction. Sequences homology $<98$, from the corresponding germline gene, were considered mutated (M-IGHV), as opposite to unmutated (U-IGHV) cases. $^{19,22}$

Cytogenetic by fluorescence in situ hybridisation (FISH) and TP53 mutations

FISH was performed on standard cytogenetic preparations from peripheral blood. ${ }^{20}$ The slides were hybridised with the multicolour probe sets LSI p53/LSI ATM and LSI D13S319/LSI 13q34/ CEP12 (Vysis-Abbott, Des Plaines, IL, USA), according to the manufacturer's protocol. ${ }^{23}$ Three hundred interphase nuclei were analysed for each probe and the cut-off for positive values were $10 \%$ for deletion of 11q22.3 (ATM), 13q14.3 (D13S319) and $17 p 13.1$ (TP53) loci and 5\% for trisomy 12. High-risk FISH refers to $11 q-$ or 17p-. As opposite, Low-risk FISH included 13q14 deletion or normal FISH. Patients harbouring trisomy 12 were considered at intermediate risk. TP53 gene sequencing between and analysis were performed according to ERIC guidelines. ${ }^{16}$

Treatment

Patients were treated accordingly to the 2008 iwCLL guidelines. ${ }^{13}$ Fludarabine or bendamustine-based regimens, with or without rituximab were used as first-line treatment in fit patients; chlorambucil with or without antiCD20 monoclonal antibody was used in elderly and/or unfit patients. FCR (fludarabine, cyclophosphamide and rituximab) ${ }^{24}$ and BR were administrated at standard doses. ${ }^{25}$ Since January 2015 patients with TP53 abnormalities were treated with BCR inhibitors, ibrutinib or idelalisib plus rituximab.

\section{Statistical analysis}

Categorical variables were compared by Chi-square test (for Binet stages and FISH) or Fisher exact's test (gender, treatment, CD38, TP53 and IGHV), when appropriate. Continuous variables (median age) were compared with Mann-Whitney test. TTFT was calculated starting from the date of diagnosis to treatment (event) or last known follow-up (censored). ${ }^{13}$ Time to next treatment (TTNT) was calculated as months from first-line FCR or BR to subsequent therapy or last known follow-up. OS were calculated starting from the date of diagnosis to death for any cause, or last known follow-up. Survival analyses were performed by the Kaplan-Meier method and the Log-rank test was used to compare survival curves between groups. Cox regression model was employed to estimate hazard ratios (HR). The cox proportional hazard assumption was assessed based on the scaled Schoenfeld residuals. The stability of our model was internally validated by bootstrap .632 method with $B=522$. The Harrell concordance index (c-index; 1.0 indicates perfect discrimination while a value 0.5 indicates equivalence to chance) was used to compare our prognostic model with Dohner's, ${ }^{23}$ FISH-IGHV and CLL-IPI models. $^{26}$ The prediction error was calculated as $1-c$-index, corrected for optimism and estimated by .632 bootstrap method. A $p$ value $>0.05$ was considered as not significant. Statistical analysis was performed with $\mathrm{R}$ (an open source statistical package downloadable from http://www.r-project.org).

\section{RESULTS}

Patients' characteristics

We gathered data from 522 CLL patients with chromosome banding analysis and IGHV status assessed within 12 months from diagnosis (Table 1). The median age at diagnosis of the whole population was 65 years, $61 \%$ were males, $76 \%$ at Binet $A$ stage, the median $\beta 2$-microglobulin was $3.27 \mathrm{mg} / \mathrm{L}, 47 \% \mathrm{U}-\mathrm{IGHV}, 9 \%$ patients harboured TP53 abnormalities and 19\% a CK. Two hundred and thirty-two patients received at least one line of therapy $(31 \%$ FCR, 16\% BR, $8 \%$ ibrutinib, 5\% chlorambucilantiCD20,40\% other treatments such as FC or chlorambucil alone, etc.) and 80 died over a median follow-up of 5.8 years. According to the subtype of CK, 30 (30\%) showed a CK1 and 69 (70\%) a CK2. In this latter group 35\% were M-IGHV and 65\% were non-mutated conformation of IGHV gene (Table 1).

As a preliminary step for our further analysis, we confirmed the established prognostic role of U-IGHV, CK and CK with major unbalanced abnormalities (i.e. CK2) in our dataset (Fig. S1A-F). The 10 -year OS was 60\% in U-IGHV and $89 \%$ M-IGHV group $(p<0.0001$, Fig. S1D); $58 \%$ for CK and $79 \%$ for no-CK patients, respectively $(p<0.0001$, Fig. S1E); $49 \%$ vs $66 \%$ vs $79 \%$ for CK2, CK1 and no-CK $(p<0.0001$, Fig. S1F), respectively. Due to the superimposable 
Table 1. Clinical and biological features of patients

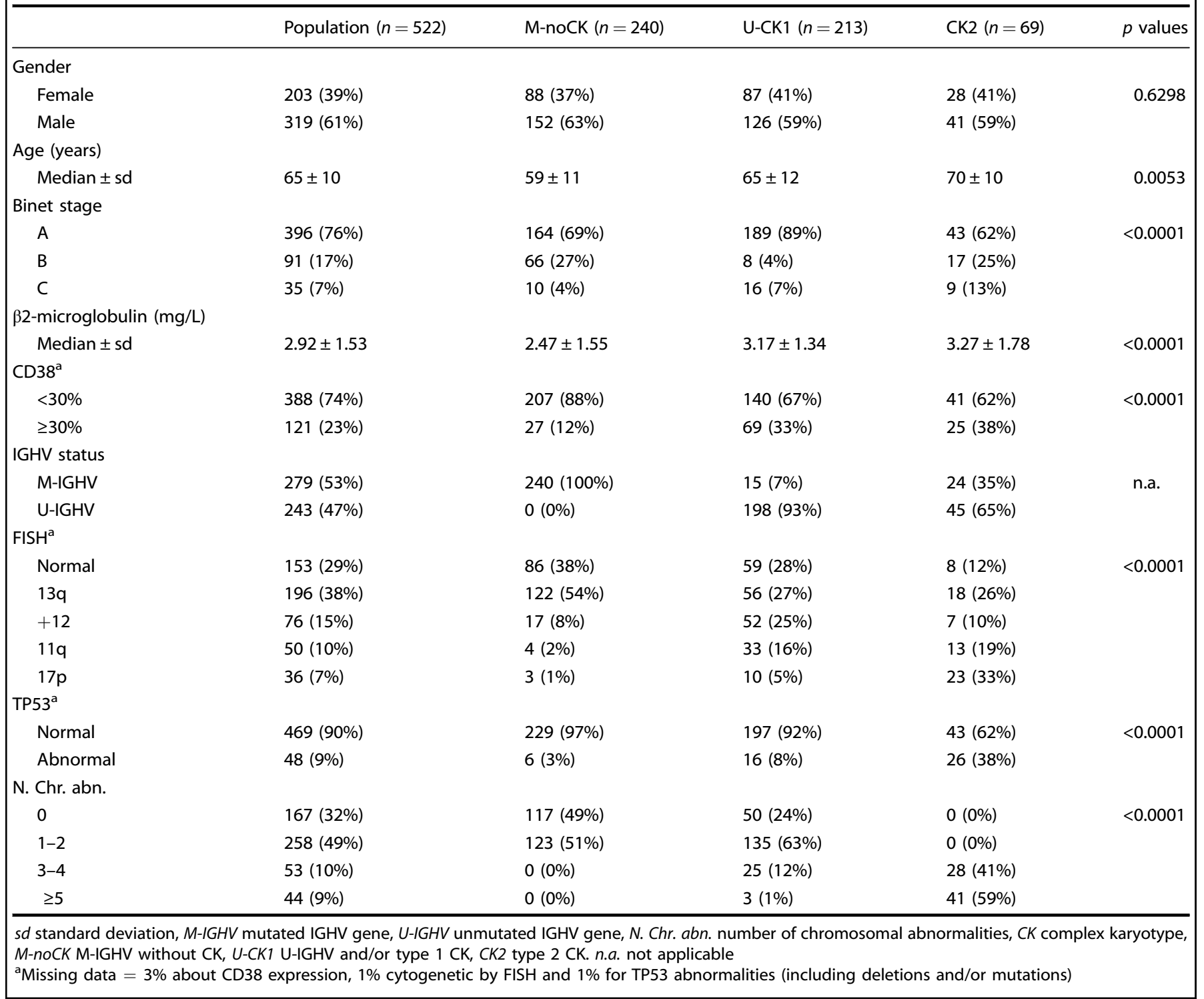

trend and absence of any statistical difference between OS curves of the CK1 and U-IGHV groups, these patients were grouped and analysed together (U-CK1), as well as M-IGHV and no-CK patients (M-noCK) (Figure S1G). Sixty-nine (13\%) patients of the whole population harboured CK2, 213 (41\%) CK1 or U-IGHV (U-CK1) and 240 (46\%) M-IGHV without any subtype of CK (M-noCK). The former group was characterised by a more advanced stage at diagnosis (Binet $C, 13 \%$ vs $7 \%$ vs $4 \%, p<0.0001$ ), higher levels of $\beta 2$-microglobulin $(2.47 \mathrm{mg} / \mathrm{L}$ vs $3.17 \mathrm{mg} / \mathrm{L}$ vs $3.27 \mathrm{mg} / \mathrm{L}$, $p<0.0001$ ), lower number of cases with low-risk FISH (i.e. 13q- or normal FISH, $38 \%$ vs $55 \%$ vs $92 \%, p<0.0001)$, but an increased prevalence of TP53 aberrations ( $38 \%$ vs $8 \%$ vs $3 \%, p<0.0001)$ and number of chromosomal abnormalities ( $\geq 5$ lesions, $0 \%$ vs $1 \%$ vs $59 \%)$, as compared to the other two groups (Table 1 ).

Prognostic impact of CK subtypes and IGHV combination We observed that patients with CK2 have a significantly shorter TTFT and OS compared to U-CK1 or M-noCK cases (Fig. 1c, d). The median OS was 7.1 years for CK2 patients but was not reached for U-CK1 and M-noCK patients $(p<0.0001)$. The 5 -year OS was $67 \%$, $85 \%$, and $93 \%$ for CK2, U-CK1 and M-noCK cases, respectively
(Fig. $1 \mathrm{~d}, p<0.0001$ ). The median and 5-year TTFT were 1.97 vs 3.40 vs 19.1 and $31 \%$ vs $39 \%$ vs $81 \%$ for CK2, U-CK 1 and M-noCK cases (Fig. 1c, $p<0.0001$ ), respectively. For both TTFT and OS, each curve was statistically different from the all others (Fig. 1c, d). Moreover, the worse prognosis of CK2 patients was independent of the TP53 (Fig. S2A-B, $p=0.0845$ and 0.8122 for TTFT and OS) and IGHV status (Fig. S2C-D, $p=0.0755$ and 0.2230 for TTFT and OS). In particular, multivariate analysis confirmed that U-CK1 patients have a 3-fold higher risk of starting treatment and of dying than M-noCK patients (Table 2, $p<0.0001$ ). Similarly, CK2 patients have 5- and 7-fold risk of undergoing first-line therapy and of dying compared to M-noCK subjects (Table 2, $p<0.0001$ ). Other variables associated with shorter TTFT and OS at univariate and multivariate analysis were advanced Binet stage, CD38+, U-IGHV, $11 q-, 17 p-$ and TP53 abnormalities (Table S1-S2). Age $>65$ years at diagnosis also predicted a shorted OS at multivariate analysis (Table S2).

Our model was also internally validated by bootstrap .632 method, showing a prediction error of 0.31 and 0.34 for TTFT and OS, respectively. Finally, the c-indexes for the FISH hierarchical model were 0.64 and 0.61 (Fig. S3A-B), the IGHV-FISH system 0.69 
a

Distribution of subgroups
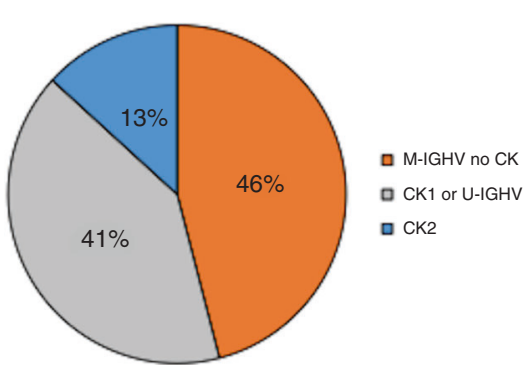

C

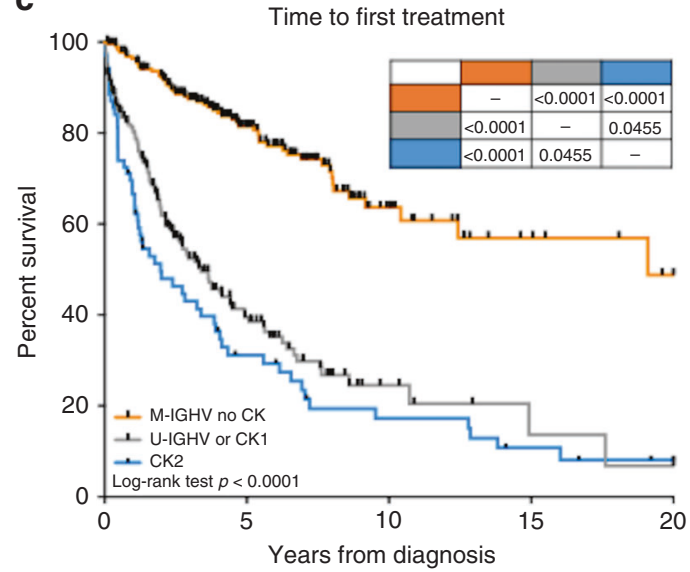

e

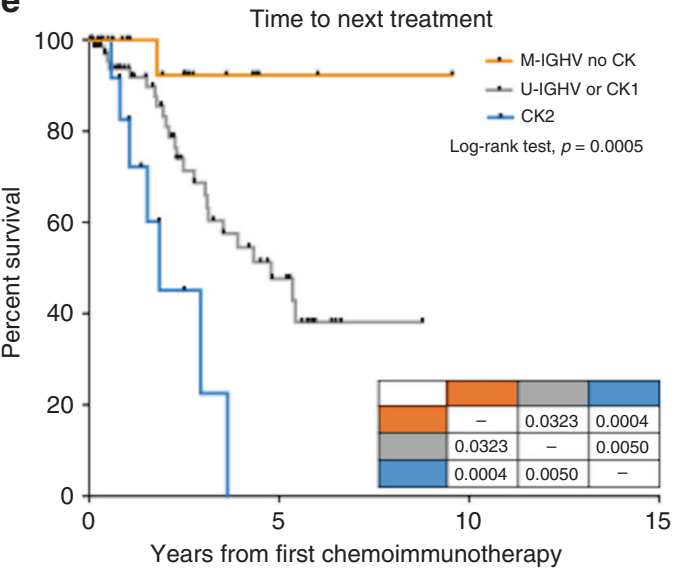

b

Frequencies of biological variables among subgroups

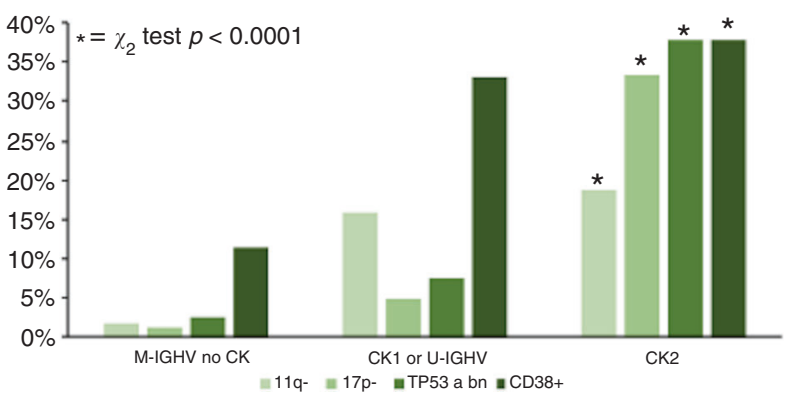

d

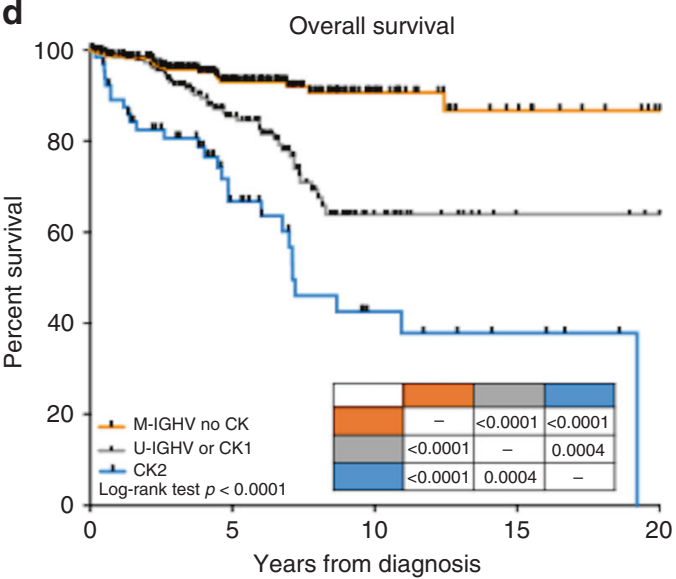

f

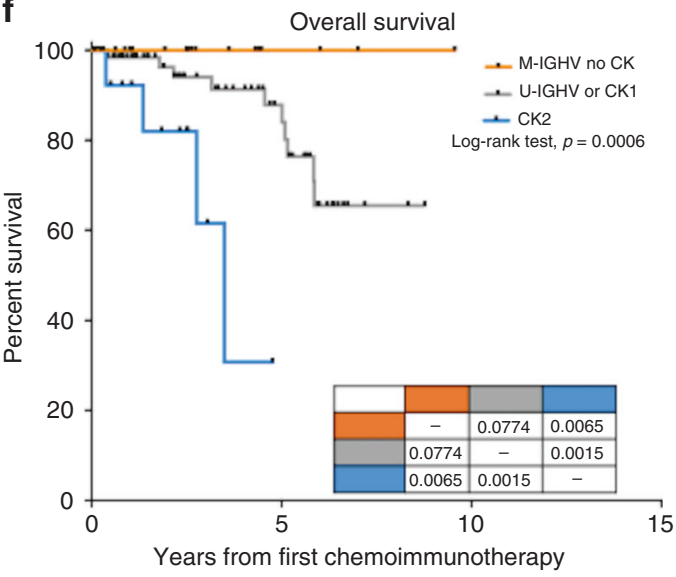

Fig. 1 In the upper-left panel a the apple-pie graph represents the distribution of patients harbouring type 2 complex karyotype (CK2, 13\%), type 1 CK or unmutated IGHV gene (U-CK1, 41\%) and mutated IGHV gene without any CK subtypes (M-noCK, 46\%). The upper-right panel b shows histograms for frequencies of biological variables among identified subgroups. CK2 subgroups was significantly enriched for CD38 $\geq$ $30 \%$ (CD38+), 11q deletion (11q-), 17p deletions (17p-), TP53 abnormalities (TP53 abn) cases compared to M-noCK or U-CK1 subjects. Panels c-f show Kaplan-Meier curves for survival analysis. Patients with CK2 (light grey curve) had the shortest time to first treatment (c) and overall survival (d) from diagnosis, as well as the worst time to next treatment (e) and overall survival (f) after chemoimmunotherapy with FCR or BR as compared with U-CK1 (dark grey curves) and M-noCK cases (black curves)

and 0.63 (Fig. S3C-D), CLL-IPI 0.67 and 0.62 (Fig. S3E-F) while for our proposed model they were 0.70 and 0.69 for TTFT and OS, respectively. These results indicate that our model was slightly better than other commonly used prognostic scores applied to our population.

Predictive impact of CK subtypes and IGHV combination The combination of the IGHV status and CK subtypes also provides predictive information after first-line therapy $(n=160$ patients, $p<$ 0.0001 for both TTNT and OS, Fig. S2G-H). In particular, focusing on 107 patients treated with FCR or BR, $20 \%$ were M-noCK, $67 \%$ U-CK1 and $13 \%$ CK2. We observed that only one of the M-noCK cases relapsed and that no patient has died after a median follow-up of 43 months as compared with the other two subgroups (Fig. 1e, f). The median TTNT was 1.86 and 4.79 years for CK2 and U-CK1, but not reached for M-noCK patients $(p<0.0005$, Fig. 1e). The estimated 3-year TTNT was 92\%, 69 and 23\%, for M-noCK, U-CK1 and CK2 patients ( $p<0.0005$, Fig. 1e), respectively. The median OS was 3.5 years for CK2 but not reached for both U-CK1 and M-noCK cases $(p=0.0006$, Fig. $1 \mathrm{f})$. The 3 -year OS was $100 \%, 94$ and $62 \%$ for M-noCK, U-CK1 and CK2 patients ( $p=0.0006$, Fig. 1f), respectively. Variables associated with TTNT and OS at univariate and 
154

\begin{tabular}{|c|c|c|c|c|c|c|}
\hline & \multicolumn{3}{|c|}{ Univariate analysis } & \multicolumn{3}{|c|}{ Multivariate analysis } \\
\hline & $\mathrm{HR}$ & $95 \%$ C.I & $p$ values & $\mathrm{HR}$ & 95\% C.I & $p$ values \\
\hline \multicolumn{7}{|l|}{ TTFT } \\
\hline M-noCK & 1.00 & - & - & 1.00 & - & - \\
\hline U-CK1 & 4.31 & $3.14-5.90$ & $<0.0001$ & 3.98 & $2.87-5.52$ & $<0.0001$ \\
\hline CK2 & 4.89 & $2.99-7.99$ & $<0.0001$ & 5.12 & $3.5-7.47$ & $<0.0001$ \\
\hline \multicolumn{7}{|l|}{ OS } \\
\hline M-noCK & 1.00 & - & - & 1.00 & - & - \\
\hline U-CK1 & 3.10 & $1.81-5.30$ & $<0.0001$ & 3.14 & $1.75-5.64$ & 0.0001 \\
\hline CK2 & 7.07 & $3.13-15.08$ & $<0.0001$ & 7.37 & $3.97-13.69$ & $<0.0001$ \\
\hline
\end{tabular}

95\% C.I. 95\% confidential interval, $M$-noCK mutated IGHV without complex karyotype, U-CK1 unmutated IGHV and/or type 1 complex karyotype, CK2 type 2 complex karyotype, TTFT time to first treatment, OS overall survival

multivariate analysis were reported in Table S3 and S4. CK2 predicted a shorter TTNT and OS also at multivariate analysis ( $p=0.0055$ and $p=0.0113$, respectively).

Subsequently, we compared CK2 patients treated with FCR or BR $(n=14)$ with those who received ibrutinib first-line $(n=7)$. Although the 3-year TTFT and OS for patients treated with chemoimmunotherapy and those with ibrutinib were $67 \%$ vs $22 \%$ and $100 \%$ vs $62 \%$, respectively, these differences were not statistically significant $(p=0.2479$ for TTNT and $p=0.2011$ for OS, Fig. S4A-B).

\section{DISCUSSION}

Chromosome banding analysis in CLL is capable of identifying chromosomal abnormalities that are missed by FISH analysis, sometimes fulfilling the criteria of $\mathrm{CK}^{4,18,27,28}$ In this retrospective study we confirmed in a large cohort of patients that CK is not a single entity but is a quantitative and qualitative cytogenetic heterogeneous category. CK patients with major structural lesions (i.e. CK2) have a dismal outcome. Furthermore, the combination of IGHV mutational status with data derived from chromosome banding analysis allows to identify a subset of patients characterised by M-IGHV without any CK subtypes with a very indolent disease, $90 \%$ alive after 10 years of follow-up, who can achieve logterm remission after a short-course of chemoimmunotherapy.

The availability of BCR- and BCL2-inhibitors, alone or in combination, is able to overcome some of the poor-risk prognostic factors associated with CLL, such as clinical stage, TP53 abnormalities and U-IGHV, and new predictive parameters are now emerging. ${ }^{1,3}$ In recent years, the prognostic and predictive role of $\mathrm{CK}$, defined by the presence of at least three chromosomal lesions, is becoming evident at diagnosis, ${ }^{4,6}$ at disease progres$\operatorname{sion}^{5}$ and in relapsed/refractory patients treated with ibrutinib ${ }^{11}$ or venetoclax. ${ }^{10}$ Although CK is found in $14-35 \%$ of CLL depending on the studies, ${ }^{4,8}$ it is a heterogeneous cytogenetic category from a quantitative and qualitative point of view. Data from the literature have documented that patients with $+12,+18$ and +19 although resembling a CK are characterised by an indolent CLL with peculiar clinical features (i.e. female predominance, young age at diagnosis, etc.); ${ }^{27}$ on the other hand the presence of at least five chromosomal aberrations predicted for a very aggressive clinical course independently of the IGHV status and TP53 lesions. ${ }^{29}$ The number of chromosomal lesions was also assessed in our study population, confirming that patients 5 or more aberrations had the shortest TTFT and OS (Fig. S5). Recently, our collaborative group has demonstrated that almost $70 \%$ of CK cases harboured major structural aberrations (such as unbalanced translocations, ring or marker chromosomes). This subset, herein called CK2, was associated with a higher incidence of TP53 aberrations, chemo-refractoriness and a shorter OS at multivariate analysis. ${ }^{12}$ Furthermore, CK2 CLL cases have a distinct mRNA expression profile with a deregulation of genes involved in cellcycle control and DNA damage response. ${ }^{12}$ In the present study we included 522 patients and we combined data derived from stimulated chromosome banding analysis with the IGHV mutational status in order to improve the prognostic and predictive power of these markers. We demonstrated that M-IGHV patients without any CK subtypes at diagnosis, corresponding to $45 \%$ of our cohort, are characterised by a very indolent disease with a median TTFT of 19 years and more $90 \%$ of them still alive after 10 years from diagnosis. Although patients with CK2 was a relatively rare subgroup, representing $13 \%$ of the cases, most of them $(81 \%)$ required a treatment within 5 years from the diagnosis, almost all needed a second line of treatment after 3 years from chemoimmunotherapy and the median OS was 7 years.

Although the exact mechanisms which favour the development of a CK are unknown, the strong association between CK and TP53 disruption herein and by other authors ${ }^{8,28,29}$ could play a relevant role. Patients with TP53 mutations are characterised by short telomers and high hTERT expression, a condition known to cause chromosome instability. ${ }^{30,31}$ Patients with TP53 disrupted showed telomere deletion and chromosomal end-to-end fusion in cells with $\mathrm{CK}^{30}$ Thomay et al. $^{32}$ reported that loss or mutation of TP53 caused an increased number of chromosomal break events leading to dicentric chromosome and whole-arm translocation. In addition, a recent paper from the German CLL Study Group found an association between short telomere length, TP53 abnormalities, early relapse after chemoimmunotherapy and adverse survival. ${ }^{33}$ In particular, cases with $17 p$ - or TP53 mutations had the shortest telomeres length, increase genomic complexity as well as clonal evolution. ${ }^{33}$

The challenge of contemporary CLL treatment involves attempts to tailor therapy according to the patients' specific biological risk profile. To responsibly and effectively advance the development of new targeted therapies, novel drugs should be specifically offered to patient subgroups who can gain the greatest benefit compared with established chemoimmunotherapy strategies. Rossi $D^{34}$ et al. demonstrated that OS of M-IGHV patients without $11 q$ or $17 p$ deletions is superimposable to that of the age-matched general population, while U-IGHV subjects and those with high-risk FISH aberrations (i.e. $11 q-$ or $17 p-$ ) invariable relapsed after FCR. This observation has been also confirmed in the re-analysis of pivotal clinical trials from the German CLL study group and MD Cancer institute. ${ }^{35,36}$ Gentile M $^{37}$ et al. published a multicentre retrospective study on $B R$ in treatment-naive patients showing that, also with this chemoimmunotherapy, M-IGHV CLL without $11 \mathrm{q} / 17 \mathrm{p}$ deletions experienced the best disease control and OS. All these observations clearly identified CLL patients with a low-risk biological profile who can achieved excellent long-term results and disease control with six cycles of chemoimmunotherapy. Our data confirm the above result ${ }^{34-36}$ and extend the observation supporting the notion that front-line chemoimmunotherapy, FCR or BR, represents a highly effective treatment option for physically fit M-IGHV CLL patients without a CK.

Given the disappointing results of CK2 patients with chemoimmunotherapy, we hypothesised that new agents, ibrutinib, would improve the outcome of this unfavourable subset. We compared CK2 patients treated with FCR or BR and those who received ibrutinib as first-line therapy. Although we found some differences between TTNT and OS curves, these were not statistically significant, likely related to the short follow-up (14 months for ibrutinib-treated patients) and the low number of patients (seven 
The combination of complex karyotype subtypes and IGHV mutational status... A Visentin et al.

CK2 cases treated with ibrutinib). The best first and subsequent therapies for patients with CK are still matter of debate. While the presence of CK has been associated to early relapse in relapsed/ refractory patients treated with ibrutinib or venetoclax, ${ }^{10,11}$ the activity of ibrutinib in treatment-naive subjects with a CK has so far not been reported. Overall the literature and current data support the importance of evaluating IGHV mutational status accordingly to recently updated iwCLL guidelines, and suggest that the outcome of CK patients with chemoimmunotherapy is disappointing due to a high rate of chemo-refractoriness, early relapse and short survival. ${ }^{5,9}$

\section{CONCLUSIONS}

Thanks to stimulated cytogenetic analysis we identified a CK in $19 \%$ of 522 CLL patients. We herein suggest that the combination of IGHV mutation and data derived from chromosome banding analysis allows to refine the prognostic stratification of CLL, to identify M-IGHV patients without any CK subtypes who are characterised by an indolent disease and excellent outcome after chemoimmunotherapy. At the other end, CK2 patients are enriched in cases with TP53 abnormalities, have unsatisfactory responses to chemotherapy and aggressive diseases with only $40 \%$ alive after 10 years of follow-up. New clinical trials incorporating a combination, or a sequence of novel agents should be envisaged for patients with CK, in particular with the CK2 subtype.

\section{AUTHOR CONTRIBUTIONS}

A.V. designed the study, performed statistical analysis, visited patients and wrote the article; S.I., E.S., S.F., M.C., E.V. and F.C. and provided intellectual inputs and visited patients; L.B., A.M., M.A.B. and M.N. performed cytogenetic tests; F.F., I.D.G., F.M. and A.G. performed cytofluorimetric and IGHV analysis; F.R.M., G.M.R., G.S., R.F., A.C. and L.T. visited patients, provided intellectual inputs and reviewed the article.

\section{ADDITIONAL INFORMATION}

Supplementary information is available for this paper at https://doi.org/10.1038/ s41416-019-0502-x.

Competing interests: A.V. received honoraria from Janssen and Abbvie. L.T. received research funding by Gilead and Janssen, advisory board for Roche, Shire and Abbvie. G.M.R. received research funding by Gilead. F.R.M. advisory board for Janssen, Shire and Abbvie. A.C. advisory board and speaker bureau for Roche, Abbvie, Gilead and Janssen. G.S. board member of Abbvie, Roche, Janssen and Celgene. R.F. advisory board or speaker bureau for Roche, Abbvie, Celgene, Incyte, Amgen, Janssen, Celtrion, Gilead and Novartis. The remaining authors declare no competing interests.

Ethical approval and consent to participate: This study was approved by the local research ethics committee of Padua hospital and informed consent was obtained from all patients.

Funding: This work was supported by funds from Associazione Italiana per la Ricerca sul Cancro (A.I.R.C.) projects to L.T. (IG-15397), Gilead fellowship program 2017 and 2018 to L.T., Special Program 'Metastatic disease: the key unmet need in oncology', AIRC $5 \times 1000$ (No. 21198) to R.F., Fondo di Ateneo per la Ricerca 2016, 2017 of the University of Ferrara to GMR and F.C., Fondo di Incentivazione alla Ricerca 2017 of the University of Ferrara to G.M.R., Ministero dell'Istruzione, dell'Università e della Ricerca PRIN 2015 to A.C. (2015ZMRFEA). A.V. received a research fellowship from the University of Padua supported by ONLUS Ricerca per Credere nella Vita (RCV), Padua, Italy.

Consent to publish: All patients gave consent to the publication of anonymous data.

Data availability: The datasets generated and analysed during the current study are not publicly available due to the data protection and lack of consent from the patients. Access to data is strictly limited to the researchers who have obtained permission for data processing.
Note: This work is published under the standard license to publish agreement. After 12 months the work will become freely available and the license terms will switch to a Creative Commons Attribution 4.0 International (CC BY 4.0).

Publisher's note: Springer Nature remains neutral with regard to jurisdictional claims in published maps and institutional affiliations.

\section{REFERENCES}

1. Scarfo, L., Ferreri, A. J. \& Ghia, P. Chronic lymphocytic leukaemia. Crit. Rev. Oncol. hematol. 104, 169-182 (2016).

2. Visentin, A., Facco, M., Frezzato, F., Castelli, M., Trimarco, V., Martini, V. et al. Integrated CLL scoring system, a new and simple index to predict time to treatment and overall survival in patients with chronic lymphocytic leukemia. Clin. Lymphoma Myeloma Leuk. 15, 612-20 e1-5 (2015).

3. Hallek, M., Cheson, B. D., Catovsky, D., Caligaris-Cappio, F., Dighiero, G., Dohner, H. et al. iwCLL guidelines for diagnosis, indications for treatment, response assessment, and supportive management of CLL. Blood 131, 2745-2760 (2018).

4. Baliakas, P., Iskas, M., Gardiner, A., Davis, Z., Plevova, K., Nguyen-Khac, F. et al. Chromosomal translocations and karyotype complexity in chronic lymphocytic leukemia: a systematic reappraisal of classic cytogenetic data. Am. J. Hematol. 89, 249-255 (2014).

5. Herling, C. D., Klaumunzer, M., Rocha, C. K., Altmuller, J., Thiele, H., Bahlo, J. et al. Complex karyotypes and KRAS and POT1 mutations impact outcome in CLL after chlorambucil-based chemotherapy or chemoimmunotherapy. Blood 128, 395-404 (2016).

6. Rigolin, G. M., Cavallari, M., Quaglia, F. M., Formigaro, L., Lista, E., Urso, A. et al. In $\mathrm{CLL}$, comorbidities and the complex karyotype are associated with an inferior outcome independently of CLL-IPI. Blood 129, 3495-3498 (2017).

7. Le Bris, Y., Struski, S., Guieze, R., Rouvellat, C., Prade, N., Troussard, X. et al. Major prognostic value of complex karyotype in addition to TP53 and IGHV mutational status in first-line chronic lymphocytic leukemia. Hematol. Oncol. 35, 664-670 (2017).

8. Rigolin, G. M., del Giudice, I., Formigaro, L., Saccenti, E., Martinelli, S., Cavallari, M. et al. Chromosome aberrations detected by conventional karyotyping using novel mitogens in chronic lymphocytic leukemia: Clinical and biologic correlations. Genes Chromosomes Cancer 54, 818-826 (2015).

9. Badoux, X. C., Keating, M. J., Wang, X., O'Brien, S. M., Ferrajoli, A., Faderl, S. et al. Fludarabine, cyclophosphamide, and rituximab chemoimmunotherapy is highly effective treatment for relapsed patients with CLL. Blood 117, 3016-3024 (2011).

10. Anderson, M. A., Tam, C., Lew, T. E., Juneja, S., Juneja, M., Westerman, D. et al. Clinicopathological features and outcomes of progression of CLL on the BCL2 inhibitor venetoclax. Blood 129, 3362-3370 (2017).

11. Thompson, P. A., O'Brien, S. M., Wierda, W. G., Ferrajoli, A., Stingo, F., Smith, S. C. et al. Complex karyotype is a stronger predictor than del(17p) for an inferior outcome in relapsed or refractory chronic lymphocytic leukemia patients treated with ibrutinib-based regimens. Cancer 121, 3612-3621 (2015).

12. Rigolin, G. M., Saccenti, E., Guardalben, E., Cavallari, M., Formigaro, L., Zagatti, B. et al. In chronic lymphocytic leukaemia with complex karyotype, major structural abnormalities identify a subset of patients with inferior outcome and distinct biological characteristics. Br. J. Haematol. 181, 229-233 (2018).

13. Hallek, M., Cheson, B. D., Catovsky, D., Caligaris-Cappio, F., Dighiero, G., Dohner, H. et al. Guidelines for the diagnosis and treatment of chronic lymphocytic leukemia: a report from the International Workshop on Chronic Lymphocytic Leukemia updating the National Cancer Institute-Working Group 1996 guidelines. Blood 111, 5446-5456 (2008).

14. Hallek, M. Chronic lymphocytic leukemia: 2015 Update on diagnosis, risk stratification, and treatment. Am. J. Hematol. 90, 446-460 (2015).

15. Langerak, A. W., Davi, F., Ghia, P., Hadzidimitriou, A., Murray, F., Potter, K. N. et al. Immunoglobulin sequence analysis and prognostication in CLL: guidelines from the ERIC review board for reliable interpretation of problematic cases. Leukemia 25, 979-984 (2011).

16. Malcikova, J., Tausch, E., Rossi, D., Sutton, L. A., Soussi, T., Zenz, T. et al. ERIC recommendations for TP53 mutation analysis in chronic lymphocytic leukemiaupdate on methodological approaches and results interpretation. Leukemia 32, 1070-1080 (2018).

17. Blanco, G., Puiggros, A., Baliakas, P., Athanasiadou, A., Garcia-Malo, M., Collado, R. et al. Karyotypic complexity rather than chromosome 8 abnormalities aggravates the outcome of chronic lymphocytic leukemia patients with TP53 aberrations. Oncotarget 7, 80916-80924 (2016).

18. Kreinitz, N., Polliack, A. \& Tadmor, T. Chronic lymphocytic leukemia is becoming more complex: how to define complex karyotype? Leuk. Lymphoma 59, 521-522 (2018). 
19. Visentin A., Facco A., Gurrieri C., Pagnin E., Martini V., Imbergamo S., et al. Prognostic and predictive impact of IGHV mutational status and load in chronic lymphocytic leukemia: focus on FCR and BR treatments. Clin. Lymphoma Myeloma Leuk. https://doi.org/10.1016/j.clml.2019.03.002 (2019).

20. Raponi, S., Del Giudice, I., Marinelli, M., Wang, J., Cafforio, L., Ilari, C. et al. Genetic landscape of ultra-stable chronic lymphocytic leukemia patients. Ann. Oncol. 29, 966-972 (2018).

21. Brochet, X., Lefranc, M. P. \& Giudicelli, V. IMGT/V-QUEST: the highly customized and integrated system for IG and TR standardized V-J and V-D-J sequence analysis. Nucleic Acids Res. 36(Web Server issue), W503-W508 (2008).

22. Hamblin, T. J., Davis, Z., Gardiner, A., Oscier, D. G. \& Stevenson, F. K. Unmutated Ig $\mathrm{V}(\mathrm{H})$ genes are associated with a more aggressive form of chronic lymphocytic leukemia. Blood 94, 1848-1854 (1999).

23. Dohner, H., Stilgenbauer, S., Benner, A., Leupolt, E., Krober, A., Bullinger, L. et al. Genomic aberrations and survival in chronic lymphocytic leukemia. N. Engl. J. Med. 343, 1910-1916 (2000).

24. Hallek, M., Fischer, K., Fingerle-Rowson, G., Fink, A. M., Busch, R., Mayer, J. et al. Addition of rituximab to fludarabine and cyclophosphamide in patients with chronic lymphocytic leukaemia: a randomised, open-label, phase 3 trial. Lancet 376, 1164-1174 (2010).

25. Eichhorst, B., Fink, A. M., Bahlo, J., Busch, R., Kovacs, G., Maurer, C. et al. First-line chemoimmunotherapy with bendamustine and rituximab versus fludarabine, cyclophosphamide, and rituximab in patients with advanced chronic lymphocytic leukaemia (CLL10): an international, open-label, randomised, phase 3, noninferiority trial. Lancet Oncol. 17, 928-942 (2016).

26. Delgado, J., Doubek, M., Baumann, T., Kotaskova, J., Molica, S., Mozas, P. et al. Chronic lymphocytic leukemia: a prognostic model comprising only two biomarkers (IGHV mutational status and FISH cytogenetics) separates patients with different outcome and simplifies the CLL-IPI. Am. J. Hematol. 92, 375-380 (2017).

27. Baliakas, P., Puiggros, A., Xochelli, A., Sutton, L. A., Nguyen-Khac, F., Gardiner, A. et al. Additional trisomies amongst patients with chronic lymphocytic leukemia carrying trisomy 12: the accompanying chromosome makes a difference. Haematologica 101, e299-e302 (2016).

28. Puiggros, A., Collado, R., Calasanz, M. J., Ortega, M., Ruiz-Xiville, N., Rivas-Delgado, A. et al. Patients with chronic lymphocytic leukemia and complex karyotype show an adverse outcome even in absence of TP53/ATM FISH deletions. Oncotarget 8 , 54297-54303 (2017)

29. Baliakas P., Jeromin S., Iskas M., Puiggros A., Plevova K., Nguyen-Khac F. et al. Cytogenetic complexity in chronic lymphocytic leukemia: definitions, associations and clinical impact. Blood 133, 1205-1216 (2019). https://doi.org/10.1182/blood2018-09-873083

30. Guieze, R., Pages, M., Veronese, L., Combes, P., Lemal, R., Gay-Bellile, M. et al. Telomere status in chronic lymphocytic leukemia with TP53 disruption. Oncotarget 7, 56976-56985 (2016).

31. Rampazzo, E., Bojnik, E., Trentin, L., Bonaldi, L., Del Bianco, P., Frezzato, F. et al. Role of miR-15a/miR-16-1 and the TP53 axis in regulating telomerase expression in chronic lymphocytic leukemia. Haematologica 102, e253-e256 (2017).

32. Thomay, K., Fedder, C., Hofmann, W., Kreipe, H., Stadler, M., Titgemeyer, J. et al. Telomere shortening, TP53 mutations and deletions in chronic lymphocytic leukemia result in increased chromosomal instability and breakpoint clustering in heterochromatic regions. Ann. Hematol. 96, 1493-1500 (2017).

33. Jebaraj B. M. C., Tausch E., Landau D. A., Bahlo J., Robrecht S., Taylor-Weiner A. N., et al. Short telomeres are associated with inferior outcome, genomic complexity, and clonal evolution in chronic lymphocytic leukemia. Leukemia 2019. https://doi. org/10.1038/s41375-019-0446-4.

34. Rossi, D., Terzi-di-Bergamo, L., De Paoli, L., Cerri, M., Ghilardi, G., Chiarenza, A. et al. Molecular prediction of durable remission after first-line fludarabinecyclophosphamide-rituximab in chronic lymphocytic leukemia. Blood 126, 1921-1924 (2015).

35. Fischer, K., Bahlo, J., Fink, A. M., Goede, V., Herling, C. D., Cramer, P. et al. Long-term remissions after FCR chemoimmunotherapy in previously untreated patients with CLL: updated results of the CLL8 trial. Blood 127, 208-215 (2016).

36. Thompson, P. A., Tam, C. S., O'Brien, S. M., Wierda, W. G., Stingo, F., Plunkett, W. et al. Fludarabine, cyclophosphamide, and rituximab treatment achieves longterm disease-free survival in IGHV-mutated chronic lymphocytic leukemia. Blood 127, 303-309 (2016).

37. Gentile, M., Shanafelt, T. D., Reda, G., Mauro, F. R., Zirlik, K., Ciolli, S. et al. Validation of a biological score to predict response in chronic lymphocytic leukemia patients treated front-line with bendamustine and rituximab. Leukemia 32, 1869-1873 (2018). 